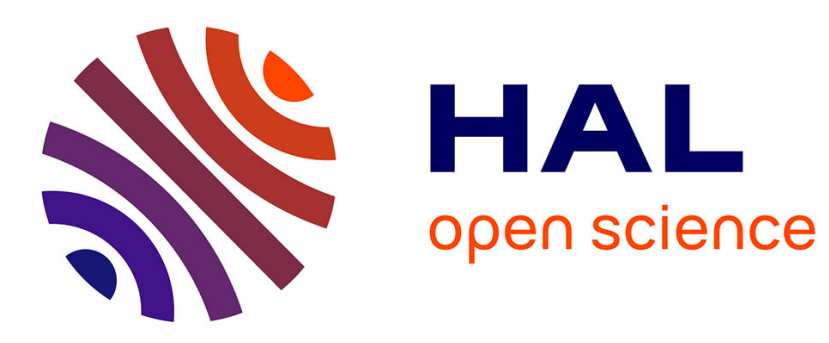

\title{
Automatic detection of pneumonia analyzing ultrasound digital images
}

Ronald H Barrientos, Avid H Roman-Gonzalez, Franklin Barrientos, Leonardo H Solis, Malena H Correa, Monica H Pajuelo, Cynthia H Anticona, Robert H Lavarello, Benjamin Castañeda, Richard H Oberhelman, et al.

\section{To cite this version:}

Ronald H Barrientos, Avid H Roman-Gonzalez, Franklin Barrientos, Leonardo H Solis, Malena H Correa, et al.. Automatic detection of pneumonia analyzing ultrasound digital images. XXXVI Convención de Centro América y Panamá - CONCAPAN 2016, Nov 2016, San Jose, Costa Rica. hal-01403814

\section{HAL Id: hal-01403814 https://hal.science/hal-01403814}

Submitted on 27 Nov 2016

HAL is a multi-disciplinary open access archive for the deposit and dissemination of scientific research documents, whether they are published or not. The documents may come from teaching and research institutions in France or abroad, or from public or private research centers.
L'archive ouverte pluridisciplinaire HAL, est destinée au dépôt et à la diffusion de documents scientifiques de niveau recherche, publiés ou non, émanant des établissements d'enseignement et de recherche français ou étrangers, des laboratoires publics ou privés. 


\title{
Automatic detection of pneumonia analyzing ultrasound digital images
}

\author{
Ronald Barrientos ${ }^{1}$, Avid Roman-Gonzalez ${ }^{1}$, Franklin Barrientos ${ }^{1}$, Leonardo Solis ${ }^{1}$, Malena Correa ${ }^{1}$, Monica \\ Pajuelo $^{1}$, Cynthia Anticona ${ }^{1}$, Roberto Lavarello², Benjamin Castañeda ${ }^{2}$, Richard Oberhelman ${ }^{3}$, William Checkley $^{4}$, \\ Robert H. Gilman ${ }^{4}$, Mirko Zimic ${ }^{1 *}$ \\ ${ }^{1}$ Laboratorio de Bioinformática y Biología Molecular, Facultad de Ciencias, Universidad Peruana Cayetano Heredia - UPCH \\ ${ }^{2}$ Laboratorio de Imágenes Médicas, Departamento de Ingeniería, Pontificia Universidad Catolica del Peru - PUCP \\ ${ }^{3}$ Tulane University, School of Public Health and Tropical Medicine - TU \\ ${ }^{4}$ Department of International Health, School of Public Health, Johns Hopkins University - JHU
}

*mirko.zimic@upch.pe

\begin{abstract}
Pneumonia is one of the major causes of child mortality. Unfortunately, in developing countries there is a lack of infrastructure and medical experts in rural areas to provide the required diagnostics opportunely. Lung ultrasound echography has proved to be an important tool to detect lung consolidates as evidence of pneumonia. This paper presents a method for automatic diagnostics of pneumonia using ultrasound imaging of the lungs. The approach presented here is based on the analysis of patterns present in rectangular segments from the ultrasound digital images. Specific features from the characteristic vectors were obtained and classified with standard neural networks. A training and testing set of positive and negative vectors were compiled. Vectors obtained from a single patient were included only in the testing or in the training set, but never in both. Our approach was able to correctly classify vectors with evidence of pneumonia, with $91.5 \%$ sensitivity and $100 \%$ specificity.
\end{abstract}

Keywords-Pneumonia, ultrasound, echography, image processing, remote diagnostics.

\section{INTRODUCTIÓN}

PNEUMONIA is the world's leading cause of child mortality, (1.4 million deaths/year) in children under five (UNICEF, 2013).

$\mathrm{X}$-ray and clinical evaluation are the most adequate tests to detect pneumonia. However, thoracic echography has also been proved to be an adequate approach to diagnose pneumonia. Unfortunately, due to its high cost, low portability, and the necessity of an expert to interpret the echographic images, the use of thoracic echography is not feasible to be used in rural areas where the presence of pneumonia is most frequent.

In this study, we show a highly sensitive and specific pattern-recognition algorithm to detect lung consolidates as evidence of pneumonia in children. The classification problem is solved in a one-layer neuralnetwork algorithm. The algorithm is evaluated on children under 5 years from the Hospital del Niño in Lima. This algorithm will permit a non-expert technician to detect pneumonia, in low-resources settings, where no specialized health care is available.

\section{METHODOLOGY}

First, we collected lung-ultrasound images from a total of 23 children under 5 years old ( 15 with diagnosis of pneumonia and 8 healthy). These children were examined at the Hospital del Niño in Lima - Peru. Lung ultrasound images (Figure 1) were obtained from a standard medical screening using a SONIXTOUCH ultrasound system with the probe L14-5/38 (Ultrasonix, Brithish Columbia, Canada). Secondly, the recorded videos were analyzed by an expert physician. Frames with clear evidence of pneumonia, as well as frames with no evidence were classified as positive or negative respectively. From these two groups of frames, characteristic vectors were extracted and classified similarly.

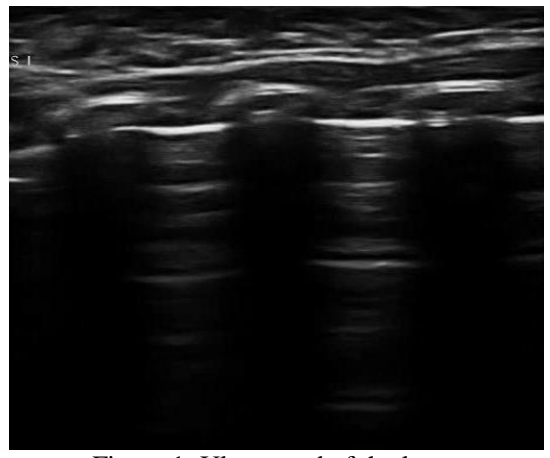

Figure 1: Ultrasound of the lung. 
Furthermore, main regions within images were identified. This procedure helped us narrowing the analysis area, and consequently reducing the amount of data and the processing time. Finally, features were extracted from the regions of interests, and fed into the neural network algorithm.

\section{A. Preliminary analysis of a lung-ultrasound image}

Due to their particular representation, specific regions within a typical lung-ultrasound image can be identified (Figure 2). First, it is noticeable, the group of pixels forming a bright and nearly-horizontal line which corresponds to the pleural line (orange). This line facilitates us to identify two main regions within the image: an upper and lower region corresponding to the skin and rib cage respectively.

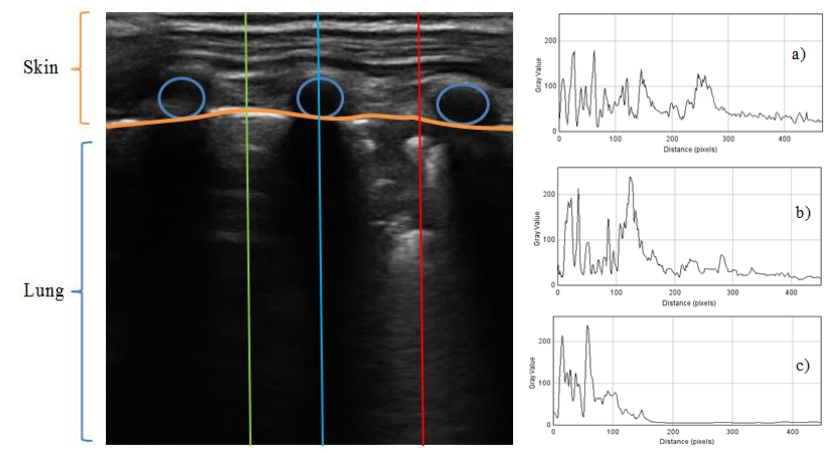

Figure 2: Echographic image where healthy (green), bone (blue), and pneumonia (red) vectors are highlighted. Brightness profile of: a) pneumonia, b) healthy, and c) bone.

The high concentration of high-value pixels in certain regions is because sound waves emitted by the ultrasound scanner are mostly reflected in highly-dense areas. Particularly, bones have a high density of matter; therefore sound waves are reflected whenever they reach the bones, generating dark areas in the ultrasound image. Specifically, ribs (blue circles) along with their shadows (located under the ribs) are depicted as relatively broad and parallel dark areas. We conveniently call these vertical areas the bone regions, as these provide criteria for discriminating areas of analysis. Similarly, the regions corresponding to intercostal spaces are visible (in terms of ultrasound imaging), and contain pixels of different intensities. These areas depict the attenuation of sound waves once they traverse the pleural line and go deeper into the human chest. Consequently, the intercostal region is the zone where our pneumonia analysis was performed.

\section{B. Brightness profile of columns}

Our unit of analysis is a vector, which stores the brightness values of a pixels column, as this helps us to discriminate the infected lung area.
Interestingly, there is an initial region which is common to most brightness profiles (Figure 2). This region contains several ups and down and follows no regular patterns at all. Besides, it contains at least one peak that in most cases reaches pixels values greater than 200. As profiles represent brightness vs. distance, and since the distance increases according to the depth in human chest, this initial region clearly corresponds to the skin. The brightness profile of the skin continues up to approximately the occurrence of the peak value, which can be inferred as the pleural-line point. From that point on, along the distance axis, characteristic patterns occur.

Looking at several brightness profiles, we can see recognizable patterns by the human eye. If this procedure is repeated, we can often notice great similarity in many of these profiles, and thus distinguish three possible vector classifications: pneumonia, healthy, and bone. First, a pneumonia vector shows a profile with mean values having an erratic behavior (Figure 2). Such profiles may present several peaks, with values comparable to those of the pleural line, and occurring beyond the pleural-line point. Second, a healthy vector shows a smooth drop in brightness, from a very pronounced maximum. From the pleural-line point, few peaks occur, and if they occur, their values are much smaller than the half of the maximum one. Finally, bone vectors show an abrupt drop in brightness from the very pronounced maximum. This corresponds to dark areas which contribute with pixels of small integer values.

Based on our just described findings, we developed a set of procedures to explore informative details of a vector; and thus collect different features from it that can be used as inputs of a neural network.

\section{Selection of the Region of Interest (RI)}

As already mentioned, the contribution in the brightness profile of the skin region is negligible. Any possible infected area is located in the region corresponding to lungs. Hence, the skin can be discarded from our analysis safely, i.e. without losing any relevant information for the purpose of pneumonia detection. Likewise, since no significant changes are present in bone regions, these are also discarded. Most pneumonia images present only one intercostal region with pneumonia evidence, while the other regions might be considered healthy. Therefore, we automatically select a new analysis region from only one intercostal region in all images, and we classified all vectors in these regions as pneumonia or healthy. Let us consider a gray scale image as a matrix, which is mathematically represented as Inxm (m rows, n columns). Moreover, every of its elements corresponds to an image pixel, whose brightness is stored as an 8-bit integer value (0: black, 255: white). Accordingly, the new analysis region is defined as a rectangle of dimension $m^{\prime} x n^{\prime}$. The red rectangle in Figure 
3 defines the Region of Interest (RI), which is manually selected.

$$
R I=\left\{I(i, j) ; \quad i_{0} \leq i \leq i_{1}=m, j_{1} \leq j \leq j_{2}\right\}
$$

Where:

$$
\begin{gathered}
i_{1}-i_{0}+1=m^{\prime} \\
j_{1}-j_{0}+1=n^{\prime}
\end{gathered}
$$

$i_{0}, i_{1}, j_{0}, j_{1}$ are selected manually for each image.

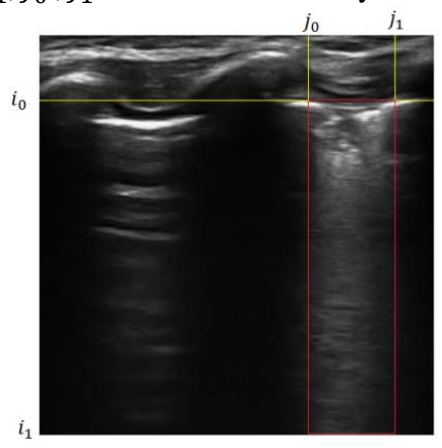

Figure 3: Selecting the analysis zone.

\section{Average brightness of " $v$ " neighbors pixels}

This operation was performed on the RI. The left-most column of pixels is initially taken as a reference. From this vector, along with its " $v-1 "$ right nearby vectors, the brightness average is calculated. In other words, each pixel is averaged with the " $v-1$ " right contiguous pixels on the same row. Similarly, this procedure was repeated for the other columns within the RI.

$$
I^{\prime}(i, j)=\frac{1}{v} \sum_{k=1}^{v} R I(i, j+k)
$$

$I^{\prime}(i, j): \quad$ is the average brightness of a RI. Its dimension is: $\left(m^{\prime}-v+1\right) \times n^{\prime}$.

\section{E. Features extraction}

To reduce the number of neurons, avoid over fitting, and to have features with more general information of the data, the following procedure is made: for each column vector (of dimension $m$ ) belonging to $I^{\prime}$, we take the average brightness of the $25,50,75$ and 100 percentile of its elements, starting from the first element towards the last one (features 1), and vice versa (features 2). The collected features are:

Features 1 (F1)

$$
\begin{gathered}
F 1_{25 \%}=\frac{1}{m^{\prime} / 4} \sum_{i=1}^{\frac{m^{\prime}}{4}} I^{\prime}(i, j) \\
F 1_{50 \%}=\frac{1}{m^{\prime} / 2} \sum_{i=1}^{m^{\prime} / 2} I^{\prime}(i, j) \\
F 1_{75 \%}=\frac{1}{3 m^{\prime} / 4} \sum_{i=1}^{\frac{3 m^{\prime}}{4}} I^{\prime}(i, j) \\
F 1_{100 \%}=\frac{1}{m^{\prime}} \sum_{i=1}^{m} I^{\prime}(i, j) \\
F 1=\left\{F 1_{25 \%}, F 1_{50 \%}, F 1_{75 \%}, F 1_{100 \%}\right\}
\end{gathered}
$$

Features 2 (F2)

$$
\begin{gathered}
F 2_{25 \%}=\frac{1}{m^{\prime} / 4} \sum_{i=\frac{3 m^{\prime}}{4}+1}^{m \prime} I^{\prime}(i, j) \\
F 2_{50 \%}=\frac{1}{m^{\prime} / 2} \sum_{i=\frac{m^{\prime}}{2}+1}^{m^{\prime}} I^{\prime}(i, j) \\
F 2_{75 \%}=\frac{1}{3 m^{\prime} / 4} \sum_{i=\frac{m^{\prime}}{4}+1}^{m^{\prime}} I^{\prime}(i, j) \\
F 2_{100 \%}=\frac{1}{m^{\prime}} \sum_{i=1}^{m^{\prime}} I^{\prime}(i, j) \\
\left.F 22_{25 \%}, F 2_{50 \%}, F 2_{75 \%}, F 2_{100 \%}\right\}
\end{gathered}
$$

\section{F. Rescaling}

The matrixes $I^{\prime}$ have different number of rows $\left(\mathrm{m}^{\prime}\right)$ because the parameters $i_{0}$ and $i_{1}$ are different in all images. Since the following steps require data of uniform size, rescaling is performed at this stage. Our rescaling consists of repeating the last pixel of every column, until all columns have $m$ rows.

$$
R(i, j)=\left\{\begin{aligned}
I^{\prime}(i, j), & i \leq m^{\prime} \\
I^{\prime}\left(m^{\prime}, j\right), & m^{\prime}<i \leq m
\end{aligned}\right.
$$

$m$ : Height of the original image

\section{G. Artificial Neural Network}

An artificial neural network is a computer learning system. It is presented as a set of layers of interconnected neurons, which exchange information between them. In fact, their connections have associated numeric weights that can be tuned based on experience, making neural networks adaptive to inputs and capable of learning. Particularly, it has the ability to make a diagnosis based on the measurable characteristics of certain phenomena. To work with a neural network, it is required to have two dichotomous databases with a finite number of characteristics. To achieve automatic detection of pneumonia patterns using neural networks, two processes are performed, namely, training and testing.

\section{Training}

We used back-propagation, a supervised learning algorithm where inputs are classified. The first database is used here to train neural networks.

First, we feed the inputs to the first layer, and then they spread through all layers, to produce an output value. If this does not match the expected result, then an error is calculated. Errors are transmitted back from the output towards the input layer. The idea is that each neuron receives a percentage of the total error, proportional to their contribution on the output result. On each iteration, the weights are optimized by decreasing the error function and making it converge. Our neural network uses sigmoid activation functions, and has three layers (input, hidden, output).

Testing 
The second database is used here to predict the data classification. Similarly as in training, we feed the input to the first layer, then they spreads through all layers, to produce an output value. Finally, we compared the predicted value with the real value, in order to test the effectiveness of our neural network.

Our analysis is focused on the $R I$, because it is more likely to find there, presence of lung consolidates, which is evidence of pneumonia. Therefore, our units of data analysis were the characteristic vectors in these regions. As a matter of fact, the sections near to the edges were discarded since these could be confused as healthy tissue. We used two classifications, "pneumonia" and "healthy" because these are the only two types of vectors found within a $R I$.

We analyzed a total of 60 ultrasound images: 30 images showed evidence of pneumonia in certain lung areas and were obtained from a total of 15 different patients. The other 30 corresponded to ultrasound images with no evidence of pneumonia; these were obtained from a total of 8 different patients. For the training set we defined the following database: 16 images showing evidence of pneumonia consolidates (corresponding to 8 patients); and 15 images with no evidence (corresponding to 4 patients). Similarly, for the testing set we defined as database: 14 images showing evidence of consolidates (corresponding to 7 patients); and 15 images with no evidence (corresponding to 4 patients). The extracted features from each database are: $F 1, F 2, R_{j}$ ( column vector of $R$ ).

\section{RESULTS ANALYSIS AND DISCUSSION}

The best classification of vectors associated to pneumonia was performed by the neural network trained with the four features $(F 1, F 2)$ explained above. In both cases the sensitivity achieved was $91.52 \%$ and $90.68 \%$ respectively, while in both cases the specificity was $100 \%$.

\begin{tabular}{|c|c|c|c|c|c|c|c|}
\hline \multicolumn{2}{|c|}{} & $\begin{array}{c}\text { Num } \\
\text { input }\end{array}$ & $v$ & $\begin{array}{c}\text { Num } \\
\text { Train }\end{array}$ & $\begin{array}{c}\text { Num } \\
\text { Test }\end{array}$ & $\begin{array}{c}\text { Sens } \\
(\%)\end{array}$ & $\begin{array}{c}\text { Spec } \\
(\%)\end{array}$ \\
\hline \multirow{2}{*}{ Features } & $\mathrm{F} 1$ & 4 & 10 & 1762 & 1293 & 91.52 & 100 \\
\cline { 2 - 8 } & $\mathrm{F} 2$ & 4 & 10 & 1762 & 1293 & 90.68 & 100 \\
\hline Rescaling & $R_{j}$ & 462 & 10 & 1762 & 1293 & 76.36 & 48.4 \\
\hline $\begin{array}{c}\text { Rescaling } \\
+ \\
\text { Features }\end{array}$ & $R_{j}$ \& F1 & 466 & 10 & 1762 & 1293 & 76.77 & 57.8 \\
\cline { 2 - 8 } & $R_{j}$ \& F2 & 466 & 10 & 1762 & 1293 & 73.57 & 50.2 \\
\hline
\end{tabular}

Table I. Performance of the trained neural networks, using different sets of input variables, including features ( $F 1$ or $F 2$ ) and the Rescaling parameters (mean intensities of the vectors)

- Num input: \# variables containing a given feature

- $\quad v$ : \# vectors neighbors we averaged

- Num Train: \# vectors used for training

- $\quad$ Num Test: \# vectors used for testing

- $\mathrm{S}(\%)$ : Sensitivity percentage

- $\quad \mathrm{E}(\%)$ : Specificity percentage

When using both the $F 1$ and $F 2$ features together with the mean intensities of the vectors, the neural network trained achieved a significantly lower sensitivity $(76.77 \%$ and $73.57 \%$ respectively), and specificity (57.8\% and $50.2 \%$ respectively). When only using the mean intensities of the vectors, the trained neural network classified the positive/negative vectors with a sensitivity of $76.36 \%$ and a specificity of $48.4 \%$ (Table I).

\section{CONCLUSIONS}

- A high sensitivity and specificity $(91.52 \%$ and $100 \%$ respectively) was achieved for the classification of characteristic vectors associated to pneumonia consolidates versus vectors with no evidence of pneumonia consolidates. This is a promising tool to further develop and improve, to be used in the automation of pneumonia diagnostics, especially in rural regions of Andean highlands where children are seriously affected by pneumonia, and diagnostics coverage is poor.

\section{Acknowledgements:}

This work was supported by NIH-1D43TW009349-03, Grand Challenge Canada 0542-01-10, Grand Challenge Canada 0688-01-10, CONCYTECFONDECYT 054-2014, PUCP-DGI 70242-2149, and 01-2013-FONDECYT.

\section{REFERENCES}

[1] UNICEF. "Pneumonia and Diarrhoea: Tackling the deadliest diseases for the world's poorest children. New York: UNICEF, 2012." (2013).

[2] M. Pereda, M. Chavez, C. Hooper-Miele, R. Gilman, M. Steinhoff, L. Checkley. "Lung Ultrasound for the Diagnosis of Pneumonia in Children: A Meta-analysis", Pediatrics, 135(4), 714-722, 2015.

[3] Xie, Jun, Yifeng Jiang, and Hung-tat Tsui. "Segmentation of kidney from ultrasound images based on texture and shape priors." Medical Imaging, IEEE Transactions on 24.1 (2005): 45-57.

[4] Gehmacher,Otto. "Ultrasound pictures of pneumonia." European Journal of Ultrasound 3.2 (1996): 161-167.

[5] Reissig, Angelika, et al. "Lung ultrasound in the diagnosis and follow-up of community-acquired pneumonia: a prospective, multicenter, diagnostic accuracy study." CHEST Journal 142.4 (2012): 965-972.

[6] Reissig, Angelika, and Roberto Copetti. "Lung ultrasound in community-acquired pneumonia and in interstitial lung diseases." Respiration 87.3 (2014): 179-189.

[7] Patrícia, S. de A., Hugo da C. Ribeiro, and Carlos Maurício C. Mendes. "Temporal trends of overall mortality and hospital morbidity due to diarrheal disease in Brazilian children younger than 5 years from 2000 to 2010." Jornal de pediatria 89.3 (2013): 315-325. 